\title{
Autophagy is deficient and inversely correlated with COX-2 expression in nasal polyps: a novel insight into the inflammation mechanism*
}

\author{
Ling-Feng Wang 1,2,\#, Chen-Yu Chien 2,3,4, Y, Yi-Hsin Yang ${ }^{5}$, Tzyh-Chyuan Hour 6 , \\ Sheau-Fang Yang ${ }^{7}$, Hau-Ren Chen ${ }^{8}$, Ke-Li Tsai ${ }^{9}$, Jenq-Yuh Ko ${ }^{10}$, Jeff Yi-Fu \\ Chen $^{11}$
}

Rhinology 53: 270-276, 2015

DOI:10.4193/Rhino 14.232

*Received for publication: September 22, 2014

Accepted: November 29, 2014

1 Department of Otolaryngology, Kaohsiung Municipal Ta-Tung Hospital, Kaohsiung, Taiwan

2 Department of Otolaryngology, Faculty of Medicine, College of Medicine, Kaohsiung Medical University, Kaohsiung, Taiwan

${ }^{3}$ Department of Otorhinolaryngology, Kaohsiung Medical University Hospital, Kaohsiung, Taiwan

${ }^{4}$ Department of Otorhinolaryngology, Kaohsiung Municipal Hsiao-Kang Hospital, Kaohsiung, Taiwan

${ }^{5}$ School of Pharmacy, College of Pharmacy, Kaohsiung Medical University, Kaohsiung, Taiwan

${ }^{6}$ Department of Biochemistry, Kaohsiung Medical University, Kaohsiung, Taiwan

${ }^{7}$ Department of Pathology, Kaohsiung Municipal Ta-Tung Hospital, Kaohsiung Medical University Hospital, Kaohsiung Medical

University, Kaohsiung, Taiwan

${ }^{8}$ Department of Life Science and Institute of Molecular Biology, National Chung Cheng University, Chia-Yi, Taiwan

${ }^{9}$ Department of Physiology, Kaohsiung Medical University, Kaohsiung, Taiwan

\# These authors contributed

${ }^{10}$ Department of Otolaryngology, National Taiwan University, College of Medicine, Taipei, Taiwan

equally to the study.

\begin{abstract}
Background: Nasal polyposis is characterised by persistent inflammation of the upper airways. Autophagy has been implicated in many chronic inflammatory diseases. Whether autophagy plays a role in nasal polyp (NP) inflammation is completely unknown and deserves investigation.
\end{abstract}

Methods: LC3 and COX-2 expression, the common autophagy and inflammation indicators, respectively, was analysed by immunoblotting in fresh tissues of NP and control nasal mucosa (NM). Primary cultures of NP-derived fibroblasts (NPDFs) and NMDFs were established for in vitro studies. Autophagy was induced by amino acid starvation and LC3 ectopic overexpression or inhibited by 3-methyladenine in the fibroblasts. Inflammation was induced by IL1- $\beta$ and TNF- $\alpha$. LC3 and COX-2 expression was confirmed in NP specimens by immunohistochemistry.

Results: LC3 expression was decreased while COX-2 expression was significantly increased in fresh NP tissues compared with the NM control. In NMDFs and NPDFs, autophagy induction by starvation and LC3 overexpression downregulated COX-2 expression. Conversely, autophagy inhibition by 3-methyladenine enhanced COX-2 expression. However, IL1- $\beta$ and TNF- $a$ had no effect on autophagy. Immunohistochemical studies on the NP specimens showed that most displayed low LC3 expression, whereas COX-2 was highly expressed in $>50 \%$ of the specimens. Examination of two consecutive NP sections from the same tissue blocks revealed a negative correlation between LC3 and COX-2 expression.

Conclusion: Autophagy is deficient in NP tissues and COX-2 is negatively regulated by autophagy in NP-derived fibroblasts. Since COX-2 is essential for the production of pro-inflammatory mediators, this study might help interpret persistent mucosal inflammation in NP. Attenuation of inflammation by restoring autophagy might be a therapeutic strategy for treating NP.

Keywords: autophagy, COX-2, inflammation, LC3, nasal polyp 


\section{Introduction}

Chronic rhinosinusitis with nasal polyps (CRSwNP) is a heterogeneous upper airway disease histologically characterised by extensive oedema and increased inflammatory cell infiltration (1). Microorganisms have been thought to contribute to the aetiology and pathophysiology of CRSwNP. However, the initiating event that triggers NP formation remains unidentified. Still, it is generally believed that abnormal and persistent host inflammation, in response to the exogenous stimuli such as microorganisms, plays a central role in the pathogenesis of CRSwNP. Supporting this are the findings that NP is typically distinguished by Th2-skewed eosinophilic inflammation with high IL-5 and eosinophil cationic protein (ECP) levels ${ }^{(2-4)}$, albeit the variation in this inflammatory profile among different ethnic entities ${ }^{(5)}$.

Cyclooxygenase-2 (COX-2) is the key enzyme that catalyzes the conversion of arachidonic acid to prostanoids, resulting in the production of prostaglandin and thromboxane mediators. Such lipid mediators are important for the generation of inflammatory response, for which COX-2 has become the target of non-steroidal anti-inflammatory drugs (NSAIDs). COX-2 is not detectable in most normal tissues but is promptly induced in response to inflammatory stimuli, leading to increased accumulation of prostanoids in inflamed tissues ${ }^{(6)}$. In cultured human NP explants, it was reported that COX-2 mRNA was spontaneously upregulated or induced by pro-inflammatory cytokines ${ }^{(7,8)}$. Likewise, a significant basal level of COX-2 mRNA was observed in NP fibroblasts, which could be further induced by inflammatory mediators ${ }^{(9)}$. Moreover, there is strong protein expression of COX-2 in chronically inflamed nasal mucosa ${ }^{(10)}$. A recent report indicated that COX-2 mRNA was upregulated in NP in patients with cystic fibrosis ${ }^{(11)}$. Therefore, COX-2 might have an impact on NP pathogenesis.

Autophagy is a cellular catabolic process that delivers cytosolic proteins and organelles to lysosomal degradation and recycling, which is necessary for diverse physiological functions such as survival, differentiation, development and homeostasis (12). However, little is known about the role of autophagy in the disease mechanism of CRSWNP. We have previously shown that autophagy is significantly deficient in NP tissues and NP-derived fibroblasts presumably due to the activation of the Akt-mTOR signaling pathway (in press). In this study, we further demonstrated that the reduction of autophagy might lead to enhanced COX-2 expression in NP tissues and NP-derived fibroblasts, suggesting a possible mechanistic link between autophagy and inflammation in NP pathogenesis.

\section{Materials and methods}

Materials and plasmid

Dulbecco's Modified Eagle's Medium (DMEM) and Hank's Ba- lanced Salt Solution (HBSS) were from Invitrogen (Carlsbad, CA, USA). Anti-microtubule-associated protein light chain 3 (LC3) antibody (LC3B, clone D11) was purchased from Cell Signaling (Danvers, MA, USA), and anti-COX-2 and anti-GAPDH were from Santa Cruz Biotechnology (Santa Cruz, CA, USA). IL-1 $\beta$, TNF- $\alpha$ and 3-Methyladenine (3-MA) were obtained from Sigma. A GFP-LC3 construct was kindly provided by Dr. W.-B. Huang of National Taiwan University, Taiwan.

\section{Patients and tissues}

The study was approved by the Institutional Review Board (IRB) of Kaohsiung Medical University Hospital. Based on the definition in the European position paper on rhinosinusitis and nasal polyps ${ }^{(13)}$, patients with latest diagnosis of CRSwNP undergoing elective endoscopic sinus surgery at our hospital were recruited. Patients with malignancies or asthma were excluded from the study. The recruited patients did not use intranasal steroid spray nor did they take other systemic medication at least two weeks before operation. Nasal polyp tissues (NP, $n=28 ; 5$ for Western blot and 23 for immunohistochemistry) were obtained from patients with CRSwNP. Control nasal mucosa (NM, $n=6$ for Western blot) was isolated from the bulla ethmoid in patients with chronic rhinosinusitis without nasal polyps (CRSsNP).

\section{Tissue lysates and Western blot analysis}

Each nasal tissue sample was washed twice with PBS and then immersed in $500 \mu \mathrm{l}$ T-PER ${ }^{\circledast}$ Tissue Protein Extraction Reagent (Thermo Scientific) with $1 \%$ protease inhibitor cocktail and $1 \%$ phosphatase inhibitor cocktail. The tissues were homogenized with a handheld Bio-Gen PRO200 homogenizer (PRO Scientific) on ice for $1 \mathrm{~min}$. Tissue lysates were collected after centrifugation at $13,000 \mathrm{rpm}$ at $4^{\circ} \mathrm{C}$ for $30 \mathrm{~min}$. Detailed procedures for Western blot analysis were previously described ${ }^{(14)}$. Briefly, tissue lysates $(60 \mu \mathrm{g})$ from each sample were separated on SDSPAGE and transferred to PVDF membrane. After blocking, the blots were incubated with antibodies against LC3, COX-2 and GAPDH. GAPDH was used as the loading control. Quantification of the data was performed by densitometry.

\section{Primary culture of nasal fibroblasts}

Nasal tissues were washed three times with antibioticscontaining Hank's Balanced Salt Solution (HBSS) and cut into small pieces $\left(1-\mathrm{mm}^{3}\right)$. The tissue explants were treated with $0.5 \%$ trypsin for $7 \mathrm{~min}$ at $37^{\circ} \mathrm{C}$ followed by neutralization with serum-containing DMEM. After washing three times with HBSS, the digested explants were plated into 6-well culture dishes (CellBIND ${ }^{\circledR}$ Surface, Corning) and cultured with DMEM containing $10 \%$ FBS in a $37^{\circ} \mathrm{C}, 5 \% \mathrm{CO}_{2}$ humidified incubator for about two weeks. Nasal fibroblasts grown to $70-80 \%$ confluence were subcultured into $10-\mathrm{cm}$ dishes for expansion. Primary cultures at the third passages were used in the following experiments. 
Induction and inhibition of autophagy

Induction of autophagy was performed by amino acid starvation and GFP-LC3 transient transfection. $1 \times 10^{5}$ nasal fibroblasts were seeded in each well of a 12-well culture dish. Following cell attachment overnight, the culture media were replaced with fresh DMEM or starvation buffer $(140 \mathrm{mM} \mathrm{NaCl}, 1 \mathrm{mM}$ $\mathrm{CaCl}_{2}, 1 \mathrm{mM} \mathrm{MgCl}{ }_{2}, 5 \mathrm{mM}$ glucose, $20 \mathrm{mM}$ Hepes, $\mathrm{pH}$ 7.4, 1\% BSA), and cell lysates were harvested at time $0 \mathrm{~h}, 3 \mathrm{~h}$ and $6 \mathrm{~h}$. For overexpression of LC3, $4 \times 10^{6}$ nasal fibroblasts in $400 \mu$ Opti$\mathrm{MEM}^{\oplus}$ medium (Invitrogen, Carlsbad, CA, USA) were transiently transfected with 5 or $20 \mu \mathrm{g}$ of GFP-LC3 construct using a BTX ECM 830 electroporator. Cell lysates were harvested $48 \mathrm{~h}$ after transfection. Inhibition of autophagy was done by treating the cells with 3-methyladenine (3-MA) (5 mM) for $24 \mathrm{~h}$. Induction or inhibition of autophagy were confirmed by Western blot analysis using anti-LC3 antibody.

\section{Immunohistochemistry}

A non-biotin, two-step polymeric detection system was used to detect COX-2 and LC3 protein expression in 23 paraffin-embedded NP sections. Briefly, the sections were deparaffinized in $x y-$ lene solution, rehydrated in gradient ethanol solutions and then antigen retrieved at $95^{\circ} \mathrm{C}$ in citrate buffer ( $\mathrm{pH} \mathrm{6.0)}$ for $30 \mathrm{~min}$. Endogenous peroxidase was blocked by incubation with 3\% hydrogen peroxide for $5 \mathrm{~min}$ at room temperature. The sections were incubated with primary antibodies (anti-COX-2 or anti-LC3; 1:100 dilution) at $37^{\circ} \mathrm{C}$ for $1 \mathrm{~h}$. After washing twice with PBS, polyDetector HRP (BioSB, Santa Barbara, CA, USA) was applied to the sections for $30 \mathrm{~min}$, followed by incubation with DAB chromogen for $2 \mathrm{~min}$ at room temperature. The samples were then counterstained by hematoxylin, dehydrated in gradient ethanol solutions and xylene and mounted. Omission of the primary antibodies served as the negative controls. Immunohistochemical results were evaluated by one blinded pathologist and two unblinded co-investigators. The intensity of immunostaining was scored based on the percentage of positively stained cells as follows: negative, $<5 \%$; weak, 5-35\%; moderate, 35-70\%; strong, $>70 \%$.

\section{Statistical analysis}

Expression of LC3 and COX- 2 in fresh nasal tissues was analysed by Student's t-test. The staining intensity of LC3 and COX-2 in NP tissue sections was analysed by Fisher's exact test. The correlation between LC3 and COX-2 expression in NP sections was further evaluated by kappa statistic (JMP 9.0, SAS Institute, Cary, NC, USA). Probability value below $0.05(p<0.05)$ was considered statistically significant.

\section{Results}

High COX-2 but low LC3 expression in fresh NP tissues Fresh tissues of six control nasal mucosa (NM) and five nasal

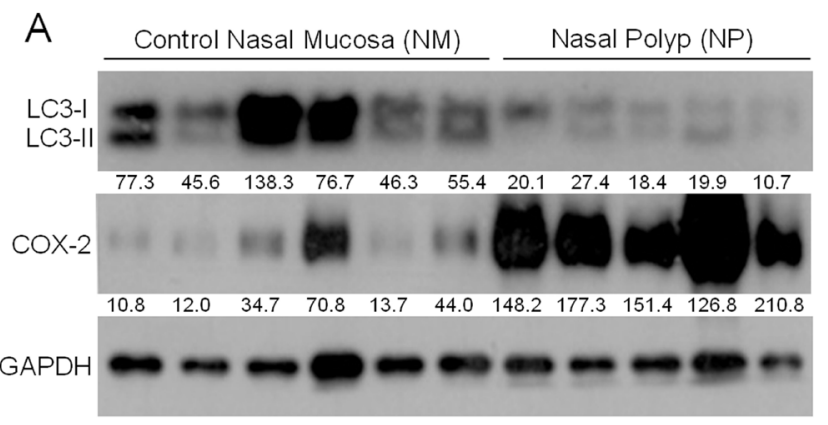

B
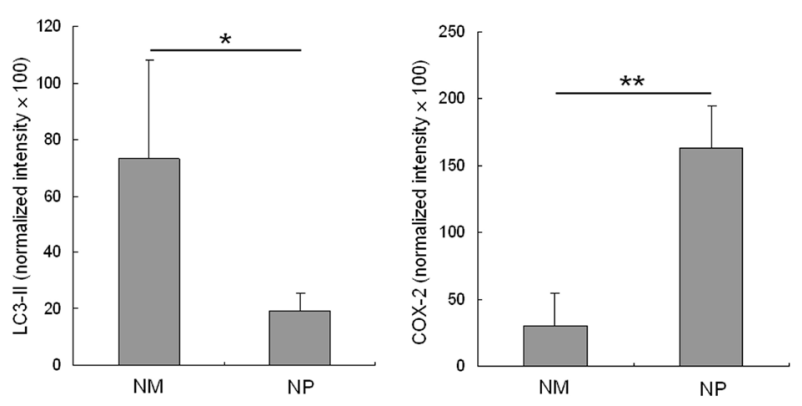

Figure 1. COX-2 was highly expressed but LC3 was nearly absent in fresh NP tissues. (A) Tissue lysates of normal nasal mucosa (NM) and NP were analysed by Western blot analysis using antibodies against LC3 and COX2. The anti-LC3 antibody recognized LC3-I, the cytosolic form of LC3, and LC3-II, the post-translational modification form of LC3-I. The amount of LC3-II was used as the indicator of autophagy. Expression of LC3 was abundant in NM but significantly diminished in NP tissues. In contrast, COX-2 expression was dramatically higher in NP than that in NM tissues. The numbers below the blots were band intensities normalized to GAPDH loading controls. (B) Quantification and statistical analysis of the results in Figure $1(A) .{ }^{*} p<0.01$ and ${ }^{* *} p<0.001$ indicate significant difference of LC3 and COX-2 expression in the nasal fibroblasts, respectively. The experiments were repeated four times, and the representative data are shown.

polyps (NP) were obtained from patients with CRSsNP and CRSwNP, respectively. Tissues lysates were isolated and then subjected to Western blot analysis for detection of the autophagy marker LC3 and inflammation marker COX-2. As shown in Figure 1, LC3 expression was much higher in NM than that in NP samples $(p<0.01)$. In sharp contrast, COX-2 expression was significantly increased in all five NP samples compared to that in the NM control $(p<0.001)$. These results indicated that autophagy was suppressed while COX-2 expression was induced in NP tissues.

\section{Autophagic activity affects COX-2 expression in NP-derived fibroblasts}

We established primary cultures of NM- and NP-derived fibroblasts (NMDFs and NPDFs) to demonstrate whether COX-2 expression was regulated by autophagy. COX-2 expression was 


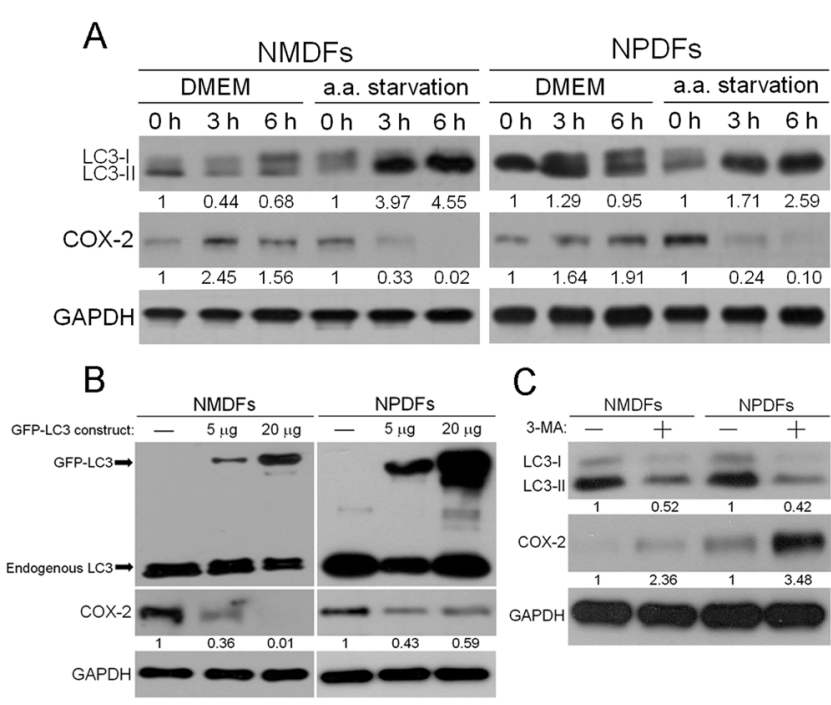

Figure 2. COX-2 was negatively regulated by autophagy. (A) NMDFs and NPDFs were incubated with the starvation buffer or DMEM for $0,3,6 \mathrm{~h}$, and induction of $\mathrm{LC} 3$ and reduction of COX-2 expression were confirmed by Western blot analysis. (B) The fibroblasts were transiently transfected with 5 or $20 \mu \mathrm{g}$ of GFP-LC3 construct. Endogenous LC3 (16 kDa) and ectopically expressed GFP-LC3 (44 kDa) were recognized by anti-LC3 antibody. COX-2 expression in the mock-transfected or transfected cells was detected by anti-COX-2 antibody. (C) The fibroblasts were treated with 3-MA (5 mM) for $24 \mathrm{~h}$ and LC3 and COX-2 expression was examined as above. The numbers below the blots were band intensity ratios of LC3-II/GAPDH or COX-2/GAPDH normalized to the untreated controls (deliberately set to 1). The experiments were repeated four times, and the representative data are presented.

decreased in a time-dependent manner when LC3 was induced by amino acid starvation for 3 or $6 \mathrm{~h}$ in both nasal fibroblasts ( $\mathrm{Fi}$ gure 2A). Consistently, induction of autophagy by forced expression of GFP-LC3 protein resulted in diminished levels of COX-2 in NMDFs and NPDFs (Figure 2B). On the other hand, suppression of autophagy by the inhibitor 3-MA ( $5 \mathrm{mM})$, as evidenced by the reduced LC3 level, led to significant upregulation of COX-2 in NPDFs (Figure 2C). These data strongly suggested that COX-2 expression was negatively regulated by autophagy especially in NPDFs.

Autophagy is unlikely to be regulated by COX-2 in NP-derived fibroblasts

To determine whether inflammation could impact autophagy in nasal fibroblasts, we examined LC3 protein level in the cells treated with inflammation inducers. NMDFs and NPDFs treated with the pro-inflammatory cytokines IL-1 $\beta$ and TNF-a (10 ng/ $\mathrm{ml}$ ) overnight significantly induced $\mathrm{COX}-2$ expression. This result also validated COX-2 as the inflammation marker for NP in the present study. However, there was no significant change of LC3 expression under the treatment of the inflammation inducers,

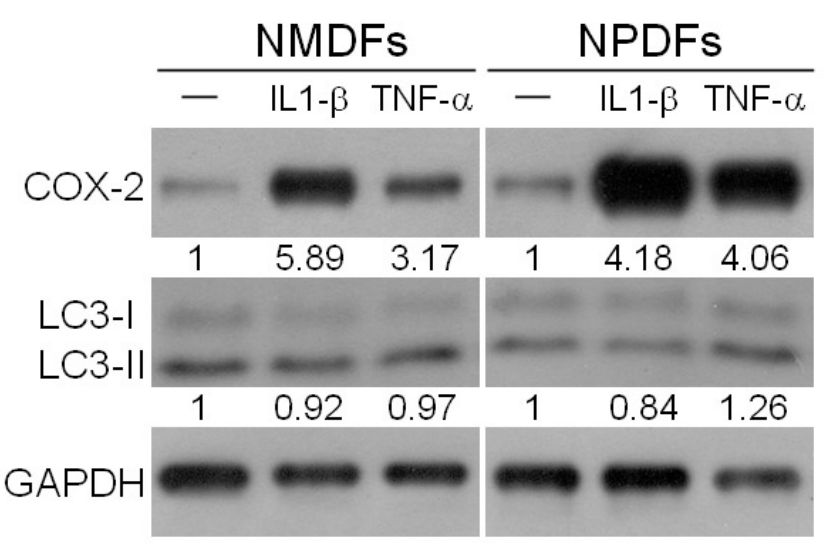

Figure 3. Induction of inflammation by pro-inflammatory cytokines did not affect LC3 expression in nasal fibroblasts. NM- and NP-derived fibroblasts (NMDFs and NPDFs) were treated with IL- $\beta$ or TNF- $\alpha$ (10 ng/ $\mathrm{ml})$, and the protein lysates were analysed by Western blot analysis using anti-LC3 and -COX-2 antibodies. COX-2 expression was significantly induced by the cytokines while LC3 level had little changes. The numbers below the blots were band intensity ratios of LC3-II/GAPDH or COX-2/ GAPDH normalized to the untreated controls (deliberately set to 1). The experiments were repeated four times, and the representative data are shown.

suggesting that the induction of COX-2 expression had little effect on autophagic activity in these nasal fibroblasts (Figure 3). These results might also rule out the possibility that inflammation exerted a negative feedback effect on autophagy.

Negative correlation between autophagy and inflammation in NP tissues by immunohistochemistry

To support the results that COX-2 expression was negatively regulated by autophagy in NP, we investigated the correlation between the expression of COX-2 and LC3 in tissue sections from 23 NP patients by immunohistochemistry. We found that the vast majority of NP tissues (17/23) had negative to weak LC3 staining intensity, while more than $50 \%$ of the NP tissues (12/23) had moderate to strong COX- 2 staining intensity $(\mathrm{p}<0.05$ by Fisher's exact test) (Table 1). Moreover, COX-2 immunostaining was localized primarily to the epithelium and moderately to the submucosal area in the NP specimens (Figure 4). Kappa statistic further demonstrated an inverse relationship between LC3 and COX-2 expression ( $\kappa=-0.363, p=0.043$ ) (Table 2). As shown in Figure 4, three representative cases of two consecutive NP sections stained respectively with anti-LC3 and anti-COX-2 antibodies revealed high COX-2 (moderate to strong) but low (or negative) LC3 expression in the same area of the tissues.

\section{Discussion}

Nasal polyps (NP) remains one of the most challenging and refractory diseases in clinical rhinology because of its hetero- 
Table 1. Immunohistochemical and statistical studies on the expression of LC3 and COX-2 in 23 NP specimens.

\begin{tabular}{lccc}
\multicolumn{4}{c}{ Staining intensity } \\
Markers & Negative + Weak & Moderate + Strong & p value* \\
\hline LC3 & 17 & 6 & 0.048 \\
COX-2 & 11 & 12 & \\
\hline
\end{tabular}

* Data were analysed by Fisher's exact test.

Table 2. Negative correlation between the expression of LC3 and COX-2 in the NP specimens.

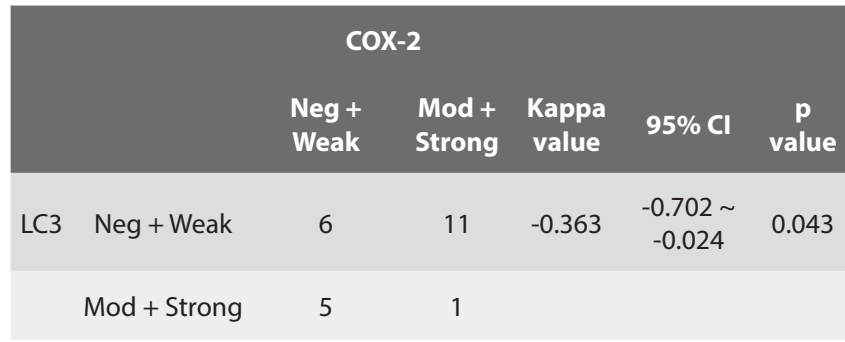

Abbreviation: Neg, negative; Mod, moderate; $\mathrm{Cl}$, confidence interval

geneous aetiology and pathophysiology and predisposition to recurrence. It occurs in about $4 \%$ of the general population, whose quality of life is seriously disturbed by the disease ${ }^{(15)}$. Unfortunately, the precise mechanism underlying the pathogenesis of NP has not been unraveled. There is an emerging consensus that persistent inflammation resulting from dysfunctional host immune response to exogenous agents contributes significantly to NP formation ${ }^{(16)}$.

Autophagy is a cellular catabolic process whereby the unwanted proteins and organelles are degraded and it has been associated with many human inflammatory diseases ${ }^{(17-20)}$. We had previously demonstrated that autophagy was suppressed in NP tissues and NP-derived fibroblasts (NPDFs) possibly due to high Akt-mTOR activity (in press). To further explore the biological function of autophagy in NP pathogenesis, we investigated the correlation between autophagy and inflammation. We found that a deficiency of autophagy was accompanied with drastically increased levels of COX-2, the widely accepted inflammation indicator, in NP tissues. In contrast, most of the control nasal mucosa (NM) had high LC3, the autophagy marker, but low COX-2 expression level. These results propelled us to hypothesize that inflammation was regulated by autophagy or vice versa in NP. The hypothesis was tested by in vitro studies. We used nasal fibroblasts as the cell model because they are the ground substances in submucosa of NP and mediate many

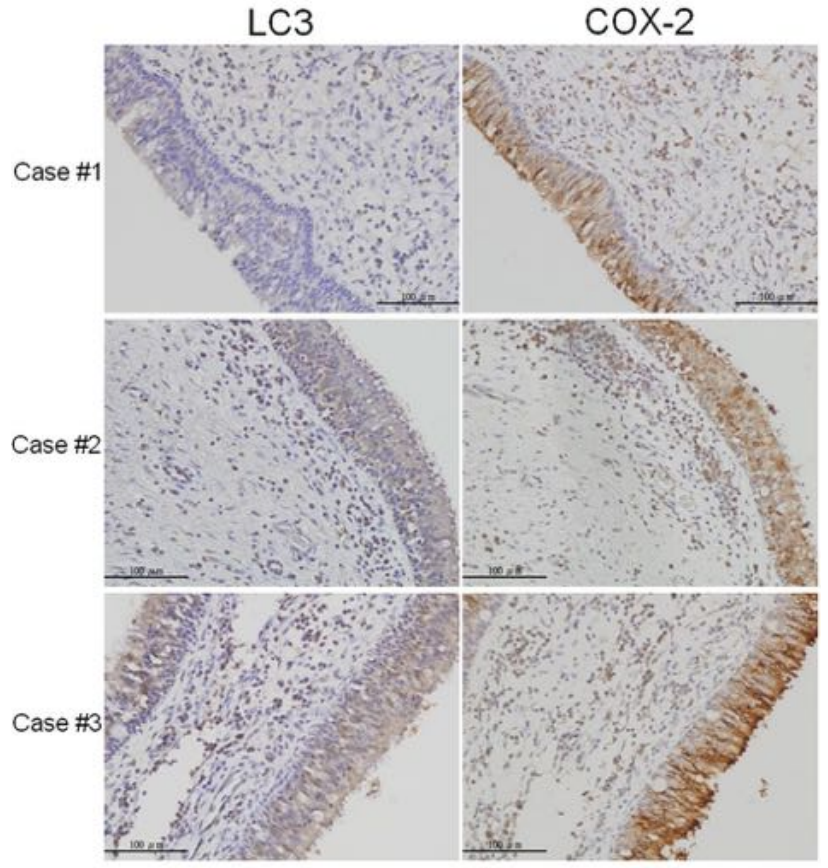

Figure 4. Immunostaining of LC3 and COX-2 in NP tissue sections. Three representative cases that display an inverse correlation between LC3 and COX-2 staining are shown. Two consecutive sections of each case were stained respectively by antibodies against LC3 and COX-2. The staining intensity of the samples was scored as followings: case \#1, strong COX-2 and negative LC3 staining; case \#2, moderate COX-2 and negative LC3 staining; case \#3, strong COX-2 and weak LC3 staining. Scale bar: 100 $\mu \mathrm{m}$. Magnification: $200 \times$

immunological responses. In addition, COX-2 was shown to be expressed in fibroblasts located in the submucosal region of NP tissues (Figure 4). By manipulating autophagic activity in this cell model, we found that COX-2 expression was decreased when autophagy was induced by amino acid starvation or LC3 ectopic overexpression. Furthermore, using the reverse approach, our data showed that COX-2 expression was increased when autophagy was attenuated by the inhibitor 3-MA in the fibroblasts. It is notable that COX-2 expression was elevated more dramatically in NPDFs than that in NMDFs despite comparable extent of LC3 reduction in the cells. This might indicate that COX- 2 was more specifically, albeit negatively, regulated by autophagy in NPDFs, consistent with the tissue results shown in Figure 1. Collectively, these results suggest that inflammation is negatively regulated by autophagy in NP, which is in line with the growing recognition that autophagy has an anti-inflammatory function that suppresses inflammasome activation ${ }^{(21-23)}$. However, the mechanism by which autophagy inhibits COX-2 expression in NP is not clear. We noticed a rapid decrease of COX-2 expression in as little as $3 \mathrm{~h}$ after autophagy induction (Figure $2 \mathrm{~A}$ ), suggesting a more likely scenario in which such repression occurred at the posttranscriptional level. There is evidence that COX-2 expres- 
sion was inhibited through modulation of its mRNA stability in cancer cells ${ }^{(24,25)}$. Whether autophagy regulates COX-2 expression by this mechanism in NP has yet to be demonstrated.

Our findings showed that COX-2 expression was negatively regulated by autophagy in NPDFs. Intriguingly, there are reports that IL-1 receptor blockade by the antagonist restores autophagy in chronic granulomatous disease (CGD) ${ }^{(18)}$ and celecoxib, a COX-2 inhibitor, induces LC3 expression in urothelial carcinoma cells ${ }^{(26)}$, indicating that inflammation might negatively regulate autophagy in certain cell contexts. To investigate this possibility in the current model, we asked whether induction of COX-2 expression could inhibit autophagy in NPDFs. Marked induction of COX-2 by the pro-inflammatory cytokines IL- $1 \beta$ and TNF- $\alpha$ had little effect on LC3 level in NPDFs. Together, it is unlikely that there is a double-negative feedback loop between autophagy and inflammation in NPDFs.

Immunohistochemical studies of 23 NP tissue sections were performed to support that autophagy was deficient and exerted an inhibitory effect on COX-2 expression in NP. Indeed, we found that autophagy, represented by LC3 expression, was mostly deficient (17/23 or $74 \%$ ) in the NP sections. In contrast, more than half of the NP sections (12/23 or 52\%) had high level of the inflammation marker COX-2. Fisher's exact test confirmed this selective downregulation of LC3 but upregulation of COX-2 in NP tissues $(p<0.05)$. To further analyse the correlation between LC3 and COX-2 expression in NP tissues, we examined the staining intensity of the two protein markers, respectively, in consecutive sections from the same patients. By kappa statistic, we showed that LC3 and COX-2 expression were negatively correlated with each other $(\kappa=-0.363, p=0.043)$. These results, in conjunction with the in vitro studies, strongly suggest that autophagy is an upstream negative regulator of inflammation in NP.

Still, it remains unknown why autophagy is deficient in NP. It has been shown that mutations in the autophagy-associated gene ATG16L1 (T300A mutation) resulted in defective autophagy activity and contributed to susceptibility to Crohn's disease ${ }^{(27,28)}$. Given our previous results that the Akt-mTOR signaling pathway is highly activated in NP tissues, it becomes less likely that the autophagy deficiency in NP was due to genetic variations. On the other hand, several lines of evidence have reported that, cystic fibrosis (CF), a chronic inflammatory airway disease, is autophagy deficient ${ }^{(29-31)}$. In these studies, a defect in the cystic fibrosis transmembrane conductance regulator (CFTR) results in autophagy deficiency because of functional sequestration of BECN1, an essential regulator for autophagosome formation, through a ROS-mediated mechanism. However, it remains to be elucidated whether it is high ROS levels that promote Akt-mTOR activity, in turn leading to inhibition of autophagy in NP.

\section{Conclusions}

In conclusion, we have demonstrated that autophagy could be involved in the mechanism of inflammation in NP. Our data indicate that autophagy is not only deficient but negatively correlated with COX-2 expression in NP. By an in vitro model, COX-2 was shown to be negatively regulated by autophagy in both NMDFs and NPDFs, raising the possibility that chronic mucosal inflammation in NP resulted from persistent deficiency of autophagy. Whether attenuating or modulating inflammation by restoring autophagy could be a plausible therapeutic strategy for NP warrants further investigation.

\section{Acknowledgements}

This study was funded by Ministry of Science and Technology (101-2314-B-037-032, 102-2314-B-037-042-MY2 and 102-2320B-037-016), health and welfare surcharge of tobacco products, Ministry of Health and Welfare (contract \# MOHW103-TDB-111-05) of the Republic of China, Kaohsiung Medical University Hospital Research Fund (KMUH101-1R37 and KMUH1022R34), Kaohsiung Municipal Ta-Tung Hospital Research Fund (KMTTH-103-002) and Chi-Kao collaboration project (101CMKMU-03 and 102CM-KMU-03 ).

\section{Author contributions}

JYFC, LFW: study concept and design; LFW, CYC, YHY, TCH, SFY: data acquisition, analysis and interpretation; JYFC: writing and drafting of the manuscript; JYFC, LFW, CYC, JYK: critical revision of the manuscript for important intellectual content; JYFC, LFW: obtaining funding; JYFC, HRC, KLT: administrative, technical and material support; JYFC: study supervision and final approval of the version to be published. All authors approved the final version of the manuscript.

\section{Conflict of interest}

None to declare.

\section{References}

1. Bateman ND, Fahy C, Woolford TJ. Nasal polyps: still more questions than answers. J Laryngol Otol. 2003; 117: 1-9.

2. Otto BA, Wenzel SE. The role of cytokines in chronic rhinosinusitis with nasal polyps. Curr Opin Otolaryngol Head Neck Surg 2008; 16: 270-274.
3. Van Bruaene N, Perez-Novo CA, Basinski TM et al. T-cell regulation in chronic paranasa sinus disease. J Allergy Clin Immunol. 2008; 121: 1435-1441, 1441.e1431-1433.

4. Van Zele T, Claeys S, Gevaert P, et al. Differentiation of chronic sinus diseases by measurement of inflammatory mediators Allergy. 2006; 61: 1280-1289.
5. Van Crombruggen K, Zhang N, Gevaert P, Tomassen P, Bachert C. Pathogenesis of chronic rhinosinusitis: inflammation. J Allergy Clin Immunol. 2011; 128: 728-732.

6. Vane JR, Bakhle YS, Botting RM. Cyclooxygenases 1 and 2. Annu Rev Pharmacol Toxicol. 1998; 38: 97-120.

7. Fernandez-Morata JC, Mullol J, Fuentes 
$M$, et al. Regulation of cyclooxygenase-1 and -2 expression in human nasal mucosa. Effects of cytokines and dexamethasone. Clin Exp Allergy. 2000; 30: 1275-1284.

8. Mullol J, Fernandez-Morata JC, Roca-Ferrer $J$, et al. Cyclooxygenase 1 and cyclooxygenase 2 expression is abnormally regulated in human nasal polyps. J Allergy Clin Immunol. 2002; 109: 824-830.

9. Liu CM, Hong CY, Shun CT, et al. Inducible cyclooxygenase and interleukin 6 gene expressions in nasal polyp fibroblasts: possible implication in the pathogenesis of nasal polyposis. Arch Otolaryngol Head Neck Surg. 2002; 128: 945-951.

10. Gosepath J, Brieger J, Mann WJ. New immu nohistologic findings on the differential role of cyclooxygenase 1 and cyclooxygenase 2 in nasal polyposis. Am J Rhinol. 2005; 19: 111-116.

11. Roca-Ferrer J, Pujols L, Gartner S, et al. Upregulation of COX-1 and COX-2 in nasal polyps in cystic fibrosis. Thorax. 2006; 61: 592-596.

12. Levine B, Kroemer G. Autophagy in the pathogenesis of disease. Cell. 2008; 132 27-42.

13. Fokkens W, Lund V, Mullol J. EP3OS 2007 European position paper on rhinosinusitis and nasal polyps 2007. A summary for otorhinolaryngologists. Rhinology. 2007; 45: 97-101.

14. Chen JY, Hung CC, Huang KL, et al. Src family kinases mediate betel quid-induced oral cancer cell motility and could be a biomarker for early invasion in oral squamous cell carcinoma. Neoplasia. 2008; 10: 1393-1401.

15. Newton JR, Ah-See KW. A review of nasal polyposis. Ther Clin Risk Manag. 2008; 4 507-512.

16. Fokkens WJ, Lund VJ, Mullol J, et al. European Position Paper on Rhinosinusitis and Nasal Polyps 2012. Rhinol Suppl. 2012; 3 p preceding table of contents, 1-298.

17. Alirezaei M, Kemball CC, Whitton JL.
Autophagy, inflammation and neurodegenerative disease. Eur J Neurosci. 2011; 33: 197-204.

18. de Luca A, Smeekens SP, Casagrande A, et al. IL-1 receptor blockade restores autophagy and reduces inflammation in chronic granulomatous disease in mice and in humans. Proc Natl Acad Sci U S A. 2014; 111: 3526-3531

19. Hall LJ, Watson AJ. Role of autophagy in NOD2-induced inflammation in Crohn's disease. Gastroenterology. 2012; 142: 1032 1034.

20. Nakahira K, Cloonan SM, Mizumura K, Choi AM, Ryter SW. Autophagy: a crucial moderator of redox balance, inflammation, and apoptosis in lung disease. Antioxid Redox Signal. 2014; 20: 474-494.

21. Nakahira K, Haspel JA, Rathinam VA, et al. Autophagy proteins regulate innate immune responses by inhibiting the release of mitochondrial DNA mediated by the NALP3 inflammasome. Nat Immunol. 2011; 12: $222-230$

22. Saitoh $T$, Fujita $N$, Jang $M H$, et al. Loss of the autophagy protein Atg16L1 enhances endotoxin-induced IL-1beta production. Nature. 2008; 456: 264-268.

23. Yoshizaki T, Kusunoki C, Kondo M, et al. Autophagy regulates inflammation in adipocytes. Biochem Biophys Res Commun. 2012; 417: 352-357.

24. Pham H, Rodriguez CE, Donald GW, et al. miR-143 decreases COX-2 mRNA stability and expression in pancreatic cancer cells. Biochem Biophys Res Commun. 2013; 439: 6-11.

25. Sun L, Zhang S, Jiang Z, et al. Triptolide inhibits COX-2 expression by regulating mRNA stability in TNF-alpha-treated A549 cells. Biochem Biophys Res Commun. 2011; 416: 99-105.

26. Huang KH, Kuo KL, Ho IL, et al. Celecoxibinduced cytotoxic effect is potentiated by inhibition of autophagy in human urothelial carcinoma cells. PLoS One. 2013; 8: e82034.

27. Choi AJ, Ryter SW. Autophagy in inflammatory diseases. Int J Cell Biol. 2011; 2011: 732798

28. Rioux JD, Xavier RJ, Taylor KD, et al. Genome-wide association study identifies new susceptibility loci for Crohn disease and implicates autophagy in disease pathogenesis. Nat Genet. 2007; 39: 596-604.

29. Luciani A, Villella VR, Esposito $S$, et al. Defective CFTR induces aggresome formation and lung inflammation in cystic fibrosis through ROS-mediated autophagy inhibition. Nat Cell Biol. 2010; 12: 863-875.

30. Luciani A, Villella VR, Esposito $S$, et al. Cystic fibrosis: a disorder with defective autophagy. Autophagy. 2011; 7: 104-106.

31. Luciani A, Villella VR, Esposito $S$, et al. Targeting autophagy as a novel strategy for facilitating the therapeutic action of potentiators on DeltaF508 cystic fibrosis transmembrane conductance regulator. Autophagy. 2012; 8: 1657-1672.

Dr. Jeff Yi-Fu Chen, PhD

Department of Biotechnology

Kaohsiung Medical University

100 Shih-Chuan 1st Road

San-Ming District

Kaohsiung 807

Taiwan

Tel: +886-7-3121101 ext. 2730

Fax: +886-7-3125339

E-mail: yifuc@kmu.edu.tw 